\title{
¿Es tan "nueva" la "Nueva Geografía Económica"? Sus aportaciones, sus límites y su relación con las políticas
}

Juan R. Cuadrado-Roura. Universidad de Alcalá - Instituto de Análisis Económico y Social, Madrid, España.

RESUMEN | La llamada Nueva Geografía Económica (NGE) ocupa actualmente un lugar relevante entre las corrientes de pensamiento en las que se apoyan los análisis territoriales. Algunas de las ideas y principios en que se basa eran ya muy conocidos por geógrafos y economistas desde hace algunas décadas, aunque los impulsores de esta corriente prácticamente los ignoraron. La principal aportación de la NGE reside en la posición que se adopta ante los temas territoriales, un intento de modelizar y encajar las interrelaciones entre economía y territorio en esquemas teóricos similares a los que utiliza el Análisis Económico. El artículo pretende contribuir a clarificar la aportación positiva que ha hecho la NGE al análisis territorial, los límites de sus planteamientos y también algunas de sus debilidades.

PALABraS ClAVE | desenvolvimiento territorial, economía regional, geografía económica.

ABSTRACT | The so-called New Economic Geography (NEG) presently has a very relevant position among the current theoretical approaches that support territorial analysis. Some of its concepts and ideas were well known by geographers and economists some decades ago, but the leaders of this approach have practically ignored their influence. The main contribution of the NEG is the position adopted to analyze territorial trends, searching to build models and to introduce the interrelations between the economic activities and the territory into theoretical approaches similar to those used by the Economic Analysis. The main objective of this paper is to clarify which are the main contributions of the NEG, but also its limits and weaknesses.

KEY WORDs | territorial development, regional economy, economic geography.

Recibido el 25 de marzo de 2013, aprobado el 13 de noviembre de 2013

E-Mail: jr.cuadrado@uah.es

Una primera versión de este texto se presentó y debatió en el Centro Regional de Investigaciones Multidisciplinares (CRIM) de la Universidad Nacional Autónoma de México (UnAM), en Cuernavaca (México), en octubre de 2011, que tenía el propósito de publicar un libro sobre la NGE. Los comentarios recibidos entonces y los posteriores de otros colegas y compańeros, en especial la profesora M. T. Gallo, han permitido mejorar dicho texto, pero, en todo caso, la responsabilidad es exclusivamente mía. 


\section{Introducción}

No cabe duda de que la Nueva Geografía Económica (NGE) ocupa hoy un lugar relevante en el ámbito de los análisis territoriales. Irrumpió con fuerza a principios de los noventa y se ha ido consolidando como un enfoque robusto e innovador en el estudio de las relaciones entre el espacio y los procesos económicos.

Las algo más de dos décadas que han transcurrido desde que las ideas germinales de la NGE vieron la luz constituyen un tiempo suficiente para poder efectuar algún tipo de valoración sobre lo que esta corriente analítica ha aportado y sus eventuales limitaciones. Es posible, sin embargo, que todavía sea necesaria una perspectiva temporal más extensa para llevar a cabo dicha valoración. Algunos autores, incluido uno de los fundadores (Krugman, 2000), pusieron ya de relieve hace algunos años que la NGE se encuentra en una "etapa de madurez", que suele ser precisamente cuando gran parte de los nuevos planteamientos y corrientes analíticas se consolidan y ofrecen sus mejores frutos. Pero también reconoce que la fase de "madurez" de cualquier movimiento científico renovador coincide, a veces, con una decreciente capacidad de innovación.

Que este sea o no el caso de la NGE debe quedar como una cuestión abierta. Sin embargo, lo que sí cabe a estas alturas es plantearse algunas preguntas sobre lo que ha significado la NGE en los estudios regionales y urbanos. La primera de tales preguntas debe ser, sin duda, si el adjetivo "nueva", que desde un principio se incorporó al sustantivo "Geografía Económica”, era y es o no adecuado y pertinente. En otras palabras: ¿es tan "nueva” la Nueva Geografía Económica?

Otras tres cuestiones pueden, o quizá deben, plantearse en relación con la NGE. La primera también resulta obligada: ¿cuáles son los puntos de partida de la NGE y qué es lo que ha marcado su desarrollo? La segunda, derivada de la anterior, es preguntarnos si la NGE ha alcanzado o no los objetivos que perseguía en sus inicios. Y, por último, si aceptamos la idea de que las ciencias sociales tienen una "clara vocación a la acción”, es decir, a no quedarse solo en el ámbito de lo abstracto o lo académico $^{1}$, algo que comparte quien suscribe este trabajo, la tercera cuestión necesaria de plantearse es: ¡cómo se ha posicionado la NGE frente a las posibles acciones o políticas para suavizar o resolver los problemas que muestra la realidad? En otras palabras: ¿está aportando la NGE ideas y propuestas de política regional, urbana, o de política económico-social?

El objetivo del artículo es ofrecer algún tipo de respuesta a estas cuatro preguntas y para ello se organiza en varias secciones que siguen a esta introducción. La segunda sección se dedica a comentar un hecho bastante conocido, como es el "olvido" del espacio por parte de los economistas y la explicación que puede darse a este hecho. La tercera describe la entrada en escena de la NGE y se plantea ofrecer una respuesta a si el adjetivo "nueva" es o no aceptable. La cuarta sección se centra en definir los puntos de partida de la NGE y la dirección de los progresos que esta corriente ha impulsado, tratando de responder a la segunda de las preguntas planteadas. La quinta sección intenta dar respuesta a la pregunta de si la NGE ha alcanzado o no los

W. Pareto (1920) subrayó ya que la práctica va con frecuencia por delante de la teoría. Pueden consultarse sobre este tema los trabajos de David (1988), Duverger (1964) o M. Bunge (1997), Kuhn (1962) o Cuadrado-Roura (2010). 
objetivos que se había propuesto. Y, por último, la sección sexta tiene como objetivo clarificar cómo se han posicionado los protagonistas vinculados a la NGE, frente la demanda de propuestas de medidas y/o políticas relativas a los problemas territoriales y económicos. El texto se cierra con unas breves notas finales que pretenden destacar las principales ideas ofrecidas. No se trata de "conclusiones", puesto que este término es demasiado rotundo y sería inadecuado establecerlas cuando la NGE está todavía in fieri, es decir, en proceso de desarrollo. Queda, pues, al mejor criterio del lector la posibilidad de extraer sus propias conclusiones, aceptando o no lo que se expone en este artículo.

\section{El olvido del territorio por parte de los economistas}

Históricamente, los economistas tendieron a menospreciar, o quizá sería mejor decir a "olvidar", o a no prestar casi ninguna atención al territorio y su relación con las actividades económicas. Esta actitud ha cambiado sustancialmente en las cuatro últimas décadas, pero hay cuando menos dos razones que contribuyeron a dicho "olvido". La primera guarda relación directa con el enfoque que fue adoptando la Economía como ciencia. La segunda tiene que ver con el divorcio que generalmente existió entre economistas y geógrafos.

\section{El desarrollo de la Ciencia Económica y el casi general olvido del espacio y de las cuestiones territoriales}

La primera de las dos razones citadas se evidencia cuando se revisa la trayectoria que ha seguido la Ciencia Económica en su desarrollo. Uno de los rasgos más claros es que, a diferencia de otras ciencias sociales, la Economía tendió casi desde un principio a minimizar el papel y la influencia del "espacio" en los procesos económicos, entendiendo como "espacio" el territorio que soporta la actividad económica y que influye en y se ve influido por los procesos de producción, circulación y distribución de los bienes, servicios, factores y rentas. Actualmente, buena parte de la literatura económica reconoce que el territorio $-\mathrm{y}$ lo que él implica- no puede quedar al margen de cualquier análisis de los hechos económicos y sociales, porque evidentemente no es "neutral". El territorio no solo dificulta o facilita los movimientos de los factores de producción y de los bienes producidos, sino que todos los agentes económicos -ya sean empresarios o simples consumidores- lo tienen en cuenta en sus decisiones. Sin embargo, esta no fue en absoluto la postura dominante en las sucesivas corrientes que dieron forma al análisis económico hasta confluir en lo que hoy es la Ciencia Económica.

Si dejamos a salvo las aportaciones de Von Thünen ${ }^{2}$ y de algunos economistas alemanes y escandinavos que se preocuparon por la localización de las actividades

2 La aportación de J. E. Von Thünen (1783-1850) constituye una excepción muy importante. Blaug (1986) señala que en él hay dos o incluso tres economistas en uno solo: "para los geógrafos económicos, es el 'padre' de la teoría de la localización (...); para los economistas teóricos, es uno de los descubridores de la teoría de la distribución (...); y para los economistas matemáticos y económetras es un pionero en la utilización del cálculo y la obtención de soluciones a los problemas de la maximización” (p. 246). 
económicas ${ }^{3}$, hay que reconocer que durante bastantes ańos ni los ya citados, ni otros temas relacionados con el territorio fueron objeto de particular atención por parte de los economistas. Los mercantilistas (desde E. Misselden, A. Serra y T. Mun hasta J. B. Colbert) y algunos fisiócratas (F. Quesnay, A-R. J. Turgot, R. Cantillon ${ }^{4}$ ) sí tuvieron en cuenta los aspectos "geográficos" de la circulación económica. Varios de ellos concedieron especial atención al comercio y la circulación, a las infraestructuras, los puertos y la distribución espacial de la población, aunque no puede afirmarse que dieran a estos temas un tratamiento sistemático. Sin embargo, esta limitada presencia de los aspectos territoriales en algunos textos preclásicos quedó muy pronto ahogada por la prioridad que los clásicos otorgaron a los problemas y cuestiones macroeconómicas y por la actitud adoptada más tarde por quienes les sucedieron.

Como es bien conocido, los economistas clásicos -desde A. Smith hasta J. S. Mill- escribieron ampliamente sobre el crecimiento económico y sus causas, sobre el comercio exterior, y sobre la distribución de la renta y los problemas fiscales, entre otros. Pero, salvo muy contadas excepciones, lo hicieron dejando a un lado la dimensión territorial y sus implicaciones; de hecho, consideraron a las economías nacionales como un todo y al espacio internacional como una constelación de "puntos" que se correspondían con los países que desarrollaban algún tipo de intercambio.

Más tarde, y ya dentro de la corriente llamada neoclásica, quienes profundizaron en el análisis microeconómico (Menger, Jevons, Walras...) aplicaron como método el análisis marginal, donde la incorporación de la dimensión espacial planteaba importantes dificultades. La variable "distancia” y otros parámetros espaciales suponían tener que admitir la existencia de "discontinuidades" (en el transporte, en la concentración de recursos y factores, o en relación con las aglomeraciones urbanas, etcétera) que resultaban bastante incómodas para razonar en términos de cálculo diferencial. El análisis teórico del comportamiento de las unidades productivas y de los consumidores permite estudiar con precisión sus posibles posiciones de equilibrio. No obstante, los costes de transporte pocas veces figuran como una variable "dentro" de la función de producción y se concede escasa o nula importancia a si los demandantes o consumidores están dispersos o concentrados en el territorio.

Si tenemos en cuenta lo anterior, el hecho de que numerosos economistas omitieran toda referencia a los aspectos espaciales, o que los considerasen -simplemente- como un factor neutro, no resulta excesivamente extraño. El Análisis Económico general avanzó, sin embargo, en amplios frentes, aunque en su mayor parte las teorías pueden ser calificadas como a-espaciales. Hay que reconocer que, en algunos temas, el hecho de no tomar en consideración el territorio no implica una grave debilidad teórica, pero en otros casos claramente sí. Sorprende, por ejemplo, que incluso en un campo tan ligado a lo espacial como es el comercio internacional se desarrollaran

3 Entre ellos, los alemanes W. Launhardt y A. Schaffle, O. Englander y A. Predöhl, o el sueco T. Palander.

4 Algunos sitúan a Cantillon fuera de la corriente fisiocrática, pero su Essai sur la nature du commerce en général, editado en 1755 (aunque ya era conocido desde 1730), relaciona de forma muy clara la producción primaria con la circulación de los bienes mediante el comercio y el papel del territorio. 
modelos en los que las distancias, los costes de transportes o las ventajas ofrecidas por determinados puntos o zonas geográficas, simplemente no figuraban en los modelos explicativos o fueron claramente infravalorados. Algo que las nuevas teorías del comercio internacional -y de ahí su vinculación a la NGE- han tratado de corregir.

Por supuesto que hay excepciones a lo que acabamos de señalar. Baste citar como referencia el hecho de que Alfred Marshall (1890), un neoclásico, concediera especial importancia a las concentraciones industriales y a las economías externas y de aglomeración, enfoque que más tarde ha sido el germen del análisis de los "distritos industriales". Y habría que citar, asimismo, a quienes exploraron los problemas de la localización industrial. Estas excepciones, sin embargo, no cambian la regla general: el territorio ha constituido, durante décadas, una variable que muy pocos economistas tomaron en consideración y que no formó parte de la mainstream de la Ciencia Económica.

\section{Los economistas y los geógrafos}

La reducida atención de los economistas a los problemas territoriales tiene una explicación complementaria que tampoco puede pasar inadvertida. El estudio de la distribución territorial de la población, la descripción de los condicionantes geográficos naturales y otras diferencias territoriales de diverso tipo se consideraron siempre el campo propio y prácticamente exclusivo de los geógrafos. "Una casta de profesionales por la que los economistas -como señaló Richardson (1975) - jamás sintieron una gran estimación” (p. 15).

Esta postura, que en parte se mantuvo durante años, ha cambiado sustancialmente en las últimas décadas. Además, era una valoración claramente injusta cuando se analizan las aportaciones desarrolladas por quienes habían trabajado en los campos de la geografía económica, la geografía humana y la geografía regional. Por el contrario, solo unos pocos economistas, en su mayoría germanos y algunos escandinavos, se preocuparon por los problemas de la localización empresarial, seguidos bastante más tarde por algunos economistas norteamericanos que, si bien hoy se consideran pioneros del análisis económico regional, como Hoover (1959), Alonso (1964) o Isard (1956), tampoco figuran en posiciones académicas y profesionales muy reconocidas, ni han formado parte de la mainstream del desarrollo de la teoría económica. Algo que también ha cambiado en los últimos años, en particular cuando algunos economistas de prestigio (R. Barro, P. Krugman y D. Quah, entre otros) han irrumpido con fuerza en el campo de los estudios regionales.

En este sentido, hay que reconocer que los desarrollos de la NGE han contribuido sustancialmente, como trataré de mostrar más adelante, a vincular Economía y Geografía. Incluso, como señalaron K. Behrens y J. F. Thisse (2007), algunos conceptos desarrollados en la NGE pueden servir y han servido para revisar varios aspectos en los que la Economía Regional había tratado de avanzar, no siempre con claridad. Este sería el caso, por ejemplo, de qué entendemos realmente como "región" y qué tipo de interrelaciones entre regiones resulta interesante estudiar, a cuyo efecto la NGE abre a economistas y geógrafos una vía sobre cómo cabe abordar estos temas. 


\section{La irrupción de la "nueva” Geografía Económica}

La entrada en escena de la NGE a principios de la década de los noventa hay que relacionarla, sin duda, con la revisión que algunos ańos antes se había hecho de la teoría del comercio internacional, que supuso la incorporación de los rendimientos crecientes, la competencia imperfecta y la posibilidad de alcanzar -al menos teóricamente- diversas posiciones de equilibrio en lo que hasta entonces habían sido los planteamientos más convencionales de la teoría del comercio internacional. A pesar de todo, como señaló Krugman (2000), “sorprendentemente, todavía tomó un largo tiempo hasta que los nuevos teóricos del comercio se dieran cuenta de las posibilidades de que la revolución en el campo de la teoría del comercio (internacional) posibilitaba hablar de cuestiones relacionadas con la Geografía Económica. El resultado fue lo que se califica [sic, en sus propias palabras] como la Nueva Geografía Económica” (p. 49-50).

Este calificativo - "nueva”- que se autoasignaron los líderes de esta corriente científica y que se ha acabado imponiendo para referirse a cuantos siguen sus enfoques, ha generado notables reacciones críticas por parte de quienes se consideran herederos de la importante corriente analítica que es la Geografía Económica, y también de bastantes estudiosos de los problemas regionales que habían surgido en el ámbito de la Economía desde mediados de los ańos cincuenta del pasado siglo 5 .

Inconscientemente, o quizá de manera muy consciente, quienes figuran como "padres" de la NGE, es decir, Krugman, Fujita y Venables, esencialmente, ignoraron lo que muchos geógrafos y bastantes economistas habían aportado al análisis regional con anterioridad. El propio Krugman ha reconocido más tarde que esto ocurrió e incluso ha pedido disculpas: "Soy consciente de que esta denominación (NGE) puede enojar a los geógrafos que tradicionalmente han trabajado duro en el estudio de la geografía económica, los cuales consideran, por una parte, que muchas de las cosas que los 'nuevos' geógrafos dicen son ya muy antiguas y, por otra, que la NGE ignora casi totalmente la realidad de los estudios realizados, como también lo hizo la teoría del comercio" (Krugman, 2000, p. 50). De ahí una pregunta clave: ¡es tan "nueva" la NGE? O esta otra menos belicosa: ¡en qué se basa la "novedad" de la NGE y qué es lo que ha aportado al conocimiento ya disponible con anterioridad?

La respuesta a ambas cuestiones puede adoptar distintos perfiles. Realmente, lo que la NGE hizo fue rescatar, en primer lugar, algunas viejas ideas y conceptos espaciales de la Economía y la Geografía Económica, particularmente las que se relacionan con la localización de las industrias y la distribución de las aglomeraciones urbanas e industriales (desde Von Thünen a Christaller, Lösch y otros). Asimismo, la NGE incorporó también, aunque sin reconocerlo abiertamente, algunos planteamientos posteriores bien conocidos en los que los problemas del desarrollo económico y su tendencia a la concentración se relacionaban directamente con el territorio, como son la tesis de la "causación circular acumulativa" (Myrdal, Hirschman...), las teorías sobre la aglomeración y las economías que ello genera (Rojas López, 2009),

5

Me refiero, en particular, a W. Isard y a quienes lo acompañaron en el desarrollo de lo que más tarde se ha calificado como "Ciencia Regional". 
las tesis centro-periferia más tradicionales y las aportaciones de quienes trabajaron en el desarrollo de la llamada "Ciencia Regional".

Como señalaron Ottaviano y Thisse (2004), muchas de las ideas de la NGE estaban presentes desde hacía mucho tiempo en los trabajos de los geógrafos económicos, y R. L. Martin (2010) no solo subraya este hecho, sino que cuestiona el alcance de las aportaciones de la NGE, algo que él y Sunley ya habían señalado con anterioridad (R. L. Martin \& Sunley, 1996). Pero ¿qué es entonces lo "nuevo"? Seguramente el principal mérito de la "nueva" aproximación a las relaciones entre procesos económicos y geografía es haber enmarcado dichas ideas dentro de un modelo de equilibrio general, lo cual ha permitido que la Geografía Económica y la teoría de la localización se incorporen a la corriente principal (mainstream) de la teoría económica.

En efecto, la principal novedad que, en mi opinión, ha aportado la NGE es que ha proporcionado un soporte analítico-matemático a muchas aportaciones que estaban ya en circulación desde hacía décadas, el cual les entrega mayor solidez y las aproxima a los criterios analíticos actualmente dominantes. Gracias a ello, los modelos y planteamientos de la NGE han encontrado un mejor acomodo dentro del Análisis Económico, al proporcionarles una mayor respetabilidad y un tipo de aproximaciones formales más rigurosas a temas como las relaciones centro-periferia, o a cómo explicar que las industrias tiendan a localizarse en un determinado punto geográfico, o la influencia que pueden ejercer algunos centros urbanos y/o determinadas infraestructuras (un puerto; un nudo ferroviario) como foco de atracción y acumulación de actividades productivas en un determinado lugar.

Sorprende, sin embargo, que la corriente de la NGE apenas hiciera referencia alguna a los antecedentes antes citados ${ }^{6}$. Tampoco lo ha hecho, por lo demás, en relación con los análisis previos sobre las economías de escala, los costes de transporte y la movilidad de los factores y sus consecuencias, conceptos básicos de la teoría económica neoclásica, que también habían sido estudiados por la Geografía teorético-cuantitativa anglosajona en las décadas de los sesenta y setenta del siglo pasado (Hagget, 1965; W. Bunge, 1966; Morril, 1970; Lloyd \& Dicken, 1972). Ni estas aportaciones, ni otras de la escuela francesa (la teoría de los "polos" de Perroux y la "polarización" de Boudeville, por ejemplo) son citadas por quienes impulsaron el desarrollo de la NGE en el panorama científico internacional ${ }^{7}$.

La constatación de estos hechos, tan innegables como sorprendentes, no puede ensombrecer, sin embargo, lo que han significado las aportaciones de la NGE en el ámbito del análisis espacial. En el caso concreto de la Economía, sus planteamientos han servido para poner fin al largo silencio con que la corriente dominante del análisis económico había tratado cuestiones tan relevantes como dónde tiene lugar la actividad económica, por qué se concentra y cuáles son las causas explicativas. Y en el ámbito de la Geografía, porque también ha estimulado un tipo de aproximaciones mucho más formalizadas y menos descriptivas en temas

6 Fujita y Krugman (1995) publicaron un trabajo - muy teórico- sobre las aportaciones de Von Thünen y de Chamberlain que pueden considerarse una excepción.

7 Bhattacharjea (2010) ha sugerido incluso que Kaldor anticipó en alguna medida los planteamientos de la NGE, o al menos algunos de sus "hallazgos". 
como la concentración de la población y de las actividades económicas. Como subrayaron Fujita, Krugman y Venables (1999):

En nuestra opinión, la cuestión que define la Geografía Económica es la necesidad de explicar las concentraciones de la población y de la actividad económica, a saber, la distinción entre los cinturones industriales y los cinturones agrícolas, la existencia de las ciudades y el papel de los núcleos industriales. En un sentido amplio, todas estas concentraciones nacen, se desarrollan y/o sobreviven debido a alguna forma de economías de aglomeración, donde la misma concentración espacial crea el ambiente económico favorable para el sostenimiento de concentraciones adicionales o continuadas (p. 14).

\section{El planteamiento de partida y los progresos de la NGE}

Aunque siempre resulta difícil y controvertido otorgar la paternidad de una determinada corriente científica y fijar su partida de nacimiento, el punto de arranque de la NGE fue muy posiblemente el artículo que Krugman publicó en 1991 sobre los rendimientos crecientes y la Geografía Económica, que constituyó una incursión bastante novedosa en el ámbito de la localización de la actividad económica. El trabajo emparentaba de alguna forma con los problemas que se había planteado Ohlin en 1933, dentro de la teoría del comercio internacional. A partir de la citada aportación, la tesis del centro-periferia y la cuestión de las aglomeraciones y su capacidad de crecimiento y de atracción, "re-descubiertos" por Krugman (1993a y 1993b), empezaron a ser objeto de otras aportaciones suyas y de quienes casi simultáneamente se habían planteado cuestiones relacionadas con la localización de la actividad económica, el uso del suelo, el desarrollo urbano, las áreas comerciales y la competencia espacial.

Fujita y Thisse (2002 y 2009) han aportado una amplia explicación sobre cómo la NGE ha ido penetrando en este campo. El principal mérito ha consistido en el "redescubrimiento" de algunos conceptos e ideas que ya estaban circulando entre los geógrafos y en algunos libros de Economía, de la mano de varios economistas más o menos marginales. Pero no solo los redescubrieron, sino que les dieron un tratamiento diferente, puesto que bastantes de dichas ideas no habían sido consideradas conjuntamente, ni sus interrelaciones se habían explorado sistemáticamente mediante modelos, lo que constituye el leit motiv, o el rasgo dominante, de la "nueva" corriente científica.

Como sugirió el propio Krugman (2000, pp. 50-51) al exponer el sentido de la NGE, la dinámica de una ciudad como Nueva York, sus condiciones más ventajosas y su desarrollo han sido muy estudiados y se conocen bastante bien apelando a los procesos de aglomeración. La estructura interna de esa o de cualquier área metropolitana puede comprenderse con relativa certeza a partir de los modelos de uso del suelo, siguiendo el enfoque pionero de Alonso (1964). No obstante, desde la óptica de la teoría del comercio internacional, este nivel de comprensión no resulta suficientemente satisfactorio. "Lo que parece necesario -sostenía Krugman en dicho trabajo- es comprender la economía de una ciudad teniendo en cuenta cómo opera 
la economía nacional -o mundial- en su conjunto. Como diría un economista, lo que uno desea es una aproximación en términos de equilibrio general".

Este tipo de enfoque debería explicar tanto los procesos de concentración como los de dispersión, y por qué se producen y cómo operan. Todo ello a partir de una visión más global, que intente explicar no solo cómo se relacionan los procesos económicos y el territorio sino también el porqué de dicha relación y sus resultados. Se trata, por tanto, de una aproximación diferente de la que podría aportar un enfoque "a la Von Thünen", que nos sugiere cómo (posiblemente) se ocupa y utiliza el territorio alrededor de una ciudad, pero que asume -como punto de partida- la existencia de ese foco central. Lo que hay que tratar de explicar, subraya Krugman (2000), son las fuerzas que conducen a la concentración o a la dispersión. Y para ello, la NGE adoptó como método el desarrollo de aproximaciones mediante modelos que permitieran comprender la dinámica de una determinada metrópoli o aglomeración en el contexto de toda la economía como conjunto. Es decir, con un enfoque de equilibrio general. A tal efecto era/es necesario tener en cuenta, de forma simultánea, las fuerzas centrípetas que tiran de la actividad económica como un todo, y las fuerzas centrífugas que la empujan hacia fuera. Y lo que hay que comprender es el juego de ambas fuerzas, teniendo en cuenta las microdecisiones de los distintos agentes que allí operan. Consecuentemente, lo primero que explora y trata de explicar la NGE es por qué las empresas y los consumidores tienden a aglomerarse en las áreas en que otras empresas y consumidores están ya localizados (Organisation for Economic Co-operation and Development [oecD], 2009).

El tema contaba ya con algunas explicaciones más o menos convincentes, como la idea de los "polos de crecimiento" que lanzó Perroux (1955), o la tesis de la causación acumulativa de Myrdal (1957), con los correspondientes efectos de atracción y de posible dispersión. Pero, de entrada, la principal aportación de la NGE radica en formalizar y profundizar sobre cómo y por qué se producen dichos mecanismos de causación acumulativa, utilizando para ello modelos que discurren desde lo más simple a su progresiva complicación. En último término, lo que esta corriente analítica ha perseguido es aportar fundamentos teóricos que permitan mostrar cómo y por qué unas regiones que son muy parecidas o incluso iguales entre sí en un punto de partida, pueden acabar diferenciándose como regiones "centro", ricas, y regiones "periféricas", que permanecen mucho más pobres.

A partir de un modelo como este, muy estilizado, los protagonistas y seguidores de la NGE han desarrollado una sucesión de modelos que responden a dicha relación centro-periferia. El más simple, que podemos incluso considerar aquí como ejemplo, partía de la teórica existencia de dos regiones parecidas, con dos sectores productivos y dos factores de producción. Es el célebre modelo del " 2 × 2 × 2", donde también se considera que cada factor es específico para un sector y que uno de los factores es móvil interregionalmente, mientras que el otro no lo es. Esto permite razonar, en abstracto, cómo operará ese "sistema" simplificado y en qué medida las economías de escala van a influir en su desarrollo. Condiciones adicionales de este modelo simplificado son que los dos bienes producidos son comercializables, si bien uno de ellos se ve dificultado por los costes "iceberg" de transporte, mientras que el otro tiene unos costes inferiores. Esto permite plantearse dos tipos de equilibrios 
estables: la aglomeración de una de las actividades productivas en una de las dos regiones, cuando los costes de transporte son bajos; y también la dispersión de dicha actividad en las dos regiones cuando los costes de transporte son elevados.

Si se toma como punto de partida un modelo tan simplificado como este, cabe irlo complicando progresivamente, como se hace en el análisis económico convencional, introduciendo las relaciones insumo-producto, o si existen o no condiciones de competencia imperfecta en ambas direcciones (Venables, 1996). Asimismo, el hecho de que -a partir de las "nuevas teorías del crecimiento" - se demuestre que las inversiones y la acumulación de capital pueden contribuir a asegurar y sostener las aglomeraciones (Baldwin, 1998), cabe analizar también los efectos de la acumulación de capital humano (Baldwin, 1999; G. I. P. Martin \& Ottaviano, 1999 y 2001) y estudiar cómo operan -al menos teóricamente- las fuerzas de aglomeración y de dispersión (Englmann \& Walz, 1995; Puga \& Venables, 1996a y 1997; Puga, 1999).

En la presentación de los "modelos" se parte siempre, como ya hemos subrayado, de ciertas simplificaciones, para introducir a continuación algunos cambios que modifiquen la situación de partida. Se busca, por ejemplo, "modelizar" lo que puede ocurrir si se producen migraciones interregionales de mano de obra, o si el número de plantas productivas se incrementa en un área concreta, o si el capital humano está muy concentrado en una sola región, con los posibles cambios y efectos que todo ello supone en el mercado. La explicación que el modelo aporta en relación con dichos cambios, siempre dentro de unos esquemas esencialmente teóricos, constituye el centro de las fuerzas de aglomeración y de dispersión de los productores, de la mano de obra y también, en último término, de los consumidores. Tales esquemas pueden contrastarse luego mediante análisis empíricos.

En este sentido, los numerosos modelos que se han elaborado por quienes han impulsado la NGE comparten algunos rasgos comunes, que pueden sintetizarse como sigue:

- Se asume que se opera en un contexto de competencia imperfecta (lo que es ya una novedad), donde los rendimientos crecientes de escala de un determinado sector económico ${ }^{9}$ constituyen la elección preferida.

- Se incorpora la idea de que los costes están directamente asociados al comercio y al transporte.

- Se pretende descubrir qué fuerzas impulsan la aglomeración (fuerzas centrípetas) o cuáles favorecen la dispersión (fuerzas centrífugas).

Dentro de las fuerzas centrípetas, casi siempre se destacan tres en particular: los movimientos migratorios de la mano de obra; las ligazones intersectoriales hacia adelante y hacia atrás entre las distintas ramas productivas (o sus empresas); y la elasticidad de la oferta de factor trabajo, vinculada al hecho de que puedan producirse transferencias de mano de obra de unas ramas productivas a otras, así como movimientos migratorios de carácter más general. Lo que podríamos calificar como

8 Es sabido que se designa con este nombre, que también se califican como "teorías del crecimiento endógeno", a las aportaciones que hicieron a partir de los ańos ochenta autores como P. Romer, R. Barro, R. Lucas, N. G. Mankiw y otros, ampliando y rectificando en alguna medida el modelo básico de R. Solow.

9 El monopolístico en el planteamiento del modelo de Dixit-Stiglitz. 
la "mecánica" del proceso de aglomeración es relativamente simple-aunque puede irse haciendo más complejo al ampliar los modelos-, ya que la mano de obra tenderá a emigrar hacia la o las regiones con una producción inicial más elevada, donde se acumulan cada vez más la producción de bienes y servicios y más puestos de trabajo. Esto incrementará la demanda, así como los beneficios y rentas locales, lo cual atrae a su vez a más empresas para producir bienes y servicios. La concentración resulta así cada vez más atractiva, al existir una amplia base de producciones intermedias que precisan muchas de las empresas allí localizadas o que se van localizando, lo cual intensifica los intercambios vía oferta y vía demanda entre todos los que producen bienes y servicios.

Uno de los temas centrales de la NGE es, como ya se ha indicado, el de las economías de aglomeración. Estas economías aumentan cuando una empresa disfruta de rendimientos crecientes de escala en un centro o región determinados, lo cual puede deberse a distintas causas. Entre ellas, la disponibilidad de recursos naturales o de localización (que no están dispersos); la posición monopolística o cuasimonopolística de una o varias empresas (condiciones de competencia imperfecta); una decisión política tomada en el pasado (por ejemplo, la decisión de que una ciudad sea la capital del país); y otras posibles razones que puedan plantearse al respecto. Los rendimientos de escala que existen atraen a su vez a más personas, que esperan obtener allí salarios más elevados, encontrarse con una mayor oferta de empleos o disfrutar de algunos beneficios laterales, como la oferta cultural o la educativa.

Este conjunto de ideas y rasgos positivos permite desplegar y tomar en consideración siete características que Baldwin, Forslid, Martin, Ottaviano y Robert-Nicoud (2003) subrayaron como alimentadoras de los distintos modelos construidos por quienes han adoptado el enfoque de la NGE:

1. El efecto del mercado propio en la concentración/dispersión.

2. La causación circular del esquema centro-periferia, al operar las fuerzas de aglomeración y de dispersión, principalmente vía migraciones.

3. La asimetría endógena (cuando las fuerzas de aglomeración dominan a las de dispersión).

4. La aglomeración catastrófica, a partir de la idea de los costes "iceberg" de Samuelson y de la rentabilidad constante del factor trabajo.

5. La superposición de expectativas y sus efectos.

6. La histéresis de localización.

7. La aglomeración/acumulación de ingresos o rentas en función de los costes, y de los impuestos, con y sin movimientos de capitales o de desindustrialización en el centro.

A partir de estas ideas, los trabajos que se han elaborado y los modelos en los que se basan han sido realmente abundantes. Tendría sin duda poco sentido detallarlos aquí, e incluso agrupándolos de acuerdo con sus afinidades su análisis sería prácticamente inabarcable. La oecd (2009), en su informe How Regions Grow, incluye 
una sistematización muy interesante y útil al respecto, aunque en estos momentos resulta ya incompleta.

En definitiva, como subrayan Fujita y Krugman (2004) al comentar el origen y los objetivos de la NGE:

La cuestión a destacar en la NGE es que trata de proporcionar alguna explicación a la formación de una gran diversidad de formas de aglomeración (o de concentración) económica en espacios geográficos. La aglomeración o agrupación (clustering) de la actividad económica tiene lugar a distintos niveles geográficos y tiene una variedad de formas distintas. Tomando un ejemplo, un tipo determinado de aglomeración surge con la agrupación de pequeñas tiendas y restaurantes en un barrio. Otro tipo de aglomeraciones lo encontramos en el proceso formación de las ciudades, donde todas adquieren distintos tamańos (...) en la emergencia de una variedad de distritos industriales; o en la existencia de fuertes desigualdades regionales dentro de un país. En el otro extremo del espectro se encuentra la estructura centro-periferia de la economía global, correspondiente al dualismo norte-sur. Es asimismo importante señalar que todos estos tipos distintos de aglomeración, a diversos niveles, están a su vez insertos en una economía mayor, formando en su conjunto un complejo sistema (p. 179).

Esta visión de dos de los fundadores de la NGE evidencia el ambicioso alcance del proyecto. Primero se intenta explicar un caso muy simple, y sus distintas variantes. Pero, a partir de ello, se procede a elevar la comprensión de lo que ocurre o puede ocurrir en un marco geográfico mucho más amplio, ya sea en un solo país, en un conjunto de países o incluso a nivel mundial. Una meta importante de la NGE es, por tanto, crear un conjunto de aproximaciones a través de modelos que permitan explicar cuestiones como la economía de una gran metrópoli (por ejemplo, Nueva York, que siempre cita Krugman), en un contexto mucho más amplio, como el propio país o el conjunto mundial.

\section{¿Ha logrado la NGE los ambiciosos objetivos que se planteó en sus inicios?}

Las posibles respuestas a esta pregunta pueden ser bastante dispares. Seguramente discurren desde una visión muy positiva sobre lo que ha significado el replanteamiento científico propuesto por la NGE, hasta duras críticas que se mueven en dos direcciones: la ignorancia que la NGE ha ejercido respecto de las aportaciones ya existentes sobre los temas que aborda; $y$, sobre todo, la presencia de un ropaje formal que proporciona rigor científico, pero que conduce a alejarse de la realidad.

Personalmente, y coincidiendo con un amplio número de investigadores interesados en los temas territoriales, estimo que la NGE ha supuesto, sobre todo, un revulsivo importante, tanto para la Geografía Económica convencional como para la Economía. Quizá mucho más para la Economía que para la Geografía, puesto que gracias al planteamiento metodológico adoptado, los temas territoriales han encontrado un espacio dentro de la Teoría Económica; un espacio que era muy reducido y casi ignorado en el pasado y que ha ganado terreno en términos de respetabilidad científica. La principal razón que justifica esto último es que el enfoque de la NGE es muy similar, si no idéntico, al que se emplea en otros campos del Análisis 
Económico. Pero dicha respetabilidad e interés se han debido también a algunas de las contribuciones realizadas en los últimos años para explicar cuestiones como la especialización productiva en relación con el territorio, sus causas y sus efectos; la utilización del suelo; el papel de las ciudades en los intercambios comerciales (a escala nacional e internacional); y la búsqueda de una visión o planteamiento más integral de las relaciones entre productos, productores, factores de producción, economías de escala y de aglomeración.

En el caso de los geógrafos, la influencia ha sido -en opinión que comparten numerosos profesionales- bastante menos marcada, sobre todo si nos referimos a quienes mantienen las posiciones más tradicionales, siempre más pegadas a la geografía y a su influencia que al análisis económico. Ello no significa, sin embargo, que no se reconozca asimismo que las aproximaciones analíticas de la NGE, generalmente mucho más abstractas que las de la Geografía tradicional, han impulsado algunos nuevos desarrollos dentro del ámbito de la Economic Geography, como muestran algunas publicaciones recientes. Entre ellas cabe citar el número especial del Journal of Economic Geography de marzo de 2011, donde los trabajos de Behrens y Robert-Nicoud, Ottaviani, Fingleton y otros, junto con los más críticos de Storper, Sheppard o R. L. Martin y Sunley, tienen extraordinario interés ${ }^{10}$.

No ha transcurrido todavía tiempo suficiente para "valorar" lo que la NGE ha tenido de "revolución científica", en el sentido expresado en su día por Thomas Kuhn (1962). Pero hay que reconocer que esta corriente ha actuado como un revulsivo en los estudios sobre las relaciones entre geografía y actividades económicas. Y tiene, además, el gran mérito de que sus planteamientos y su metodología están en línea con el enfoque dominante en las ciencias sociales, basado en la formulación de modelos, la abstracción y su aplicación posterior a posibles casos reales. Con todo, la euforia con que al principio se acogieron las ideas y propuestas de la NGE ha ido dejando paso a algunas críticas que permiten cuestionarse su futuro desarrollo o que se considere que algunas líneas exploradas en los últimos ańos no son ya particularmente innovadoras.

La investigación económica dominante tiende a caracterizarse por un ciclo expansión-caída; cuando surge un nuevo conjunto de ideas, tiene lugar una ola de entusiasmo debido a que emergen nuevas fronteras de investigación, pero más tarde surgen sentimientos de desafección cuando los límites de las nuevas ideas se hacen patentes. La NGE ha seguido, de alguna forma, este proceso. En los primeros años, los economistas se mostraron entusiasmados por el hecho de que una importante área -los estudios regionales-, que había permanecido bastante marginada, se abría a una forma sistemática de pensar, y algunos geógrafos más tradicionales tuvieron asimismo la sensación de que su campo estaba recibiendo finalmente la atención que merecía. Sin embargo, se ha puesto de manifiesto que "mientras los modelos de la nueva geografía contribuyeron por primera vez a introducir las consideraciones espaciales en modelos suficientemente rigurosos para entrar a formar parte del canon analítico, dichos modelos son demasiado simples, demasiado estilizados para representar o reproducir bien la geografía económica del mundo real. Para quienes

Varios de estos escritos figuran entre las referencias utilizadas para preparar este trabajo. 
en cualquier caso son escépticos en relación con la modelización formal -un grupo en el que se incluyen muchos geógrafos- el hecho de que se revelen los límites de los modelos puede confirmar su opinión” (Krugman, 2000, pp. 58-59).

Esta cita evidencia la percepción que parecía empezar a darse en torno a la NGE, en boca de uno de sus fundadores: cierta pérdida de su capacidad innovadora. Como él y otros reconocen, una de las principales causas del problema y de muchas críticas es la tentación de trabajar en lo que resulta más fácil de "modelizar", en lugar de lo que parece más importante en la práctica. Además, una crítica bastante común es que muchos problemas y procesos ligados a las relaciones economía/territorio se caracterizan por su complejidad, lo cual choca con un tipo de aproximaciones mucho más simplificadas y teóricas. Alfred Marshall (1890) sugirió, por ejemplo, que existían al menos tres razones importantes para la localización de las industrias: lo que actualmente consideramos como relaciones intersectoriales (de demanda y de oferta); el papel de la especialización laboral y sus reducidos mercados; y la difusión tecnológica. Frente a ello, muchos modelos de la NGE solo tienen en cuenta uno de estos factores, lo que genera modelos unidimensionales o en los que solo se consideran dos posibles localizaciones alternativas, claramente alejados del mundo real, aunque permiten un alto grado de sofisticación teórica.

¿Significa lo anterior que la NGE ha hecho -está haciendo- una aportación de carácter "menor" al conocimiento, tratamiento y explicación de las cuestiones territoriales en relación con los procesos económicos? La respuesta que doy a esta pregunta es negativa. El progreso del análisis científico requiere la introducción de simplificaciones, que permitan "aproximar" algún tipo de explicación -siempre parcial- a una realidad que es, ante todo y sobre todo, "compleja". La construcción de modelos -particularmente en el ámbito de lo económico-implica siempre realizar simplificaciones, introduciendo supuestos y dejando a un lado determinados aspectos para centrarse en aquel o aquellos que un modelo puede permitir encajar y comprender mejor ${ }^{11}$. Consecuentemente, desde este punto de vista, el enfoque básico de la NGE es correcto y se inscribe en una línea que es común a la metodología de avance científico que tiene lugar en otras áreas del conocimiento.

El problema no radica en la utilidad y/o la validez de este procedimiento científico "simplificador", que ha probado ya su utilidad para explicar los comportamientos teóricos de los agentes económicos, al menos en el campo de la Economía ${ }^{12}$. El problema se produce cuando se magnifican dichos modelos simplificados, cuando se "olvidan" en exceso los supuestos adoptados, o cuando no quiere advertirse que hay o puede haber causas y factores que se han excluido del análisis, los cuales no solo son parte de la realidad que se quiere explicar, sino que pueden llegar a ser clave para comprender dicha realidad.

¿Cabe deducir de lo expuesto que la NGE se aparta esencialmente de la "realidad"? En una interesante reflexión, Storper (2011) afirma que, en último término, la NGE

11 J. Schlefer ha publicado recientemente (2012) un libro muy interesante sobre el papel de los supuestos en lo que hacen los economistas. Lleva por título, precisamente, The Assumptions Economists Make (Cambridge, ma y Londres: The Belknap Press of Harvard University Press).

12 Piénsese, por ejemplo, en la teoría del consumidor y su equilibrio parcial; o en los modelos que relacionan las funciones de costes de una empresa -siempre en abstracto- con los ingresos. 
busca explicar la concentración espacial de la actividad económica. Y lo hace considerando que es algo endógeno al proceso económico y, por tanto, que no depende de la "primera naturaleza geográfica", como la desigual distribución de los recursos naturales, el clima o la proximidad a las costas o a los ríos. Las economías de escala inducen la concentración de trabajadores y empresas, todos quieren disfrutar de un contacto lo más próximo posible a los mercados y del acceso a la más amplia variedad de inputs y productos. En este sentido, los costes del comercio se integran totalmente en esta vía de análisis, lo cual no se hacía previamente. Y esos costes de los intercambios pueden tener también características endógenas, puesto que las interacciones a nivel local pueden dar lugar a mayores economías de escala y a diferencias importantes (un gap) en los costes del comercio entre los agentes económicos locales y los que están más lejos.

En los planteamientos básicos de la NGE no falta, realmente, un deseo de aproximación a la realidad. Conectan, en alguna medida, con lo que ya había preocupado a autores como Myrdal, Furtado y Prebisch, o, más o menos directamente, con las aportaciones de Leontief y otros. Todos ellos subrayaron que la realidad económica mostraba características que conducían a generar desigualdades espaciales mediante procesos de autoalimentación y de aglomeración de carácter acumulativo. Un buen número de las aportaciones de la NGE ha buscado "comprender" los procesos que conducen a la aglomeración. Para ello se ha elegido el camino de los modelos y la formalización, que sin duda acaban confiriendo a bastantes de sus aportaciones un

perfil muy teórico y de carácter microeconómico, pero esto no impide -ni debería impedir- el estudio de casos reales. De hecho, bastantes aportaciones recientes se inscriben en esta última línea, aunque hay que reconocer que la tónica dominante es la de trabajos mucho más académicos que prácticos.

\section{La NGE, ¿̨ha tenido implicaciones en términos de políticas territoriales o ha inducido "nuevas" políticas?}

De lo expuesto en los apartados anteriores puede deducirse que las ideas desarrolladas por la NGE sobre la concentración espacial de la actividad económica y los posibles factores que la provocan constituyen su aportación más destacada. Se distancian de las tesis que defienden los equilibrios económicos, que propone el enfoque neoclásico, y refuerzan la hipótesis de que las fuerzas del mercado conducen $-\mathrm{o}$ pueden conducir- a que las disparidades entre regiones o aglomeraciones urbanas se agraven.

Aunque su aproximación es predominantemente "teórica", una de las razones que han acercado a la realidad las aportaciones basadas en la NGE ha sido la introducción de supuestos más realistas que los de algunas teorías convencionales. Este es el caso, por ejemplo, de admitir la existencia de condiciones de competencia imperfecta, o de aceptar que algunas ventajas están "localizadas" (los recursos naturales, los beneficios que otorga una determinada posición geográfica o política) y/o la movilidad de los factores, en particular la mano de obra) en busca de mejores condiciones y una mayor rentabilidad, etcétera. Todo ello permite comprender que las dinámicas de concentración benefician a unas áreas en relación con otras, que acaban siendo 
menos desarrolladas o se deprimen, lo cual al parecer debería conducir, inexorablemente, a defender la conveniencia de poner en práctica algún tipo de politicas orientadas a "reequilibrar" las relaciones entre unas y otras regiones.

Este último aspecto no ha sido, desde luego, el que más ha preocupado a la mayor parte de los protagonistas de la NGE. Las ciencias sociales tienen una clara vocación a la "praxis", a la puesta en práctica de actuaciones que corrijan o detengan los procesos que no se consideran socialmente aceptables (Cuadrado-Roura, 2010). Aunque se pregona que el científico no debe transcurrir del análisis teórico a las recomendaciones políticas - como prescribe la "regla de Hume"-, lo cierto es que una amplísima mayoría de las aportaciones analíticas que han tenido lugar en el campo de la Economía, la Sociología, la Geografía y otras ciencias sociales, han derivado hacia recomendaciones y propuestas de política económica y social por parte de sus autores. Esto ocurrió ya en Adam Smith y David Ricardo, pero la línea de continuidad de este planteamiento alcanza hasta Pigou, Keynes o Lucas, por no citar a otros muchos autores, tanto clásicos como contemporáneos. De hecho, Myrdal (1953) demostró hace muchos ańos cuál había sido la conducta de los economistas más destacados en relación con la introducción de juicios de valor y/o de propuestas políticas como colofón a sus teorías. La conclusión general de su análisis fue rotunda: salvo escasas excepciones, todos los economistas transgredieron la línea que debería separar la teoría y la praxis o las recomendaciones.

Pues bien, la idea de hacer propuestas operativas de política económica general o de política regional y urbana está escasamente presente -hasta ahora- en los trabajos de la NGE. De hecho, el enfoque metodológico adoptado ha conducido a "distanciarse" de la preocupación por realizar propuestas en relación con los procesos de concentración. Aparentemente, lo que puedan hacer las regiones o aglomeraciones "perdedoras" tampoco preocupa a la mayor parte de quienes han desarrollado distintos aspectos del modelo centro-periferia. La tendencia dominante ha sido mantenerse en el ámbito de lo teórico. R. L. Martin y Sunley (1996) van incluso más allá, al afirmar que la NGE no solo no es tan "nueva" como se pretendía, sino que tampoco es Geografía, porque contenía de hecho muy poco "espacio" y demasiadas matemáticas.

En este sentido, los geógrafos de la escuela teórico-cuantitativa ya habían aceptado que los modelos teóricos y el formalismo matemático los alejaban del complejo mundo real. Esto hizo que se inclinasen hacia el análisis de las interacciones espaciosociedad y al estudio de las diversas formas que adquiere la territorialidad en el desarrollo económico, tratando de extraer de todo ello consecuencias e ideas para posibles actuaciones.

Quienes lideran o han liderado ${ }^{13}$ la NGE reconocieron hace bastante tiempo que el material publicado en relación con ese campo contenía escasos esfuerzos en cuanto

13 Esta afirmación no es gratuita. Desde que obtuvo el Nobel de Economía, P. Krugman prácticamente no ha realizado ninguna nueva aportación en el ámbito de la NGE, pero tampoco muchas en el campo donde fue asimismo pionero, el del Comercio Internacional. Las conferencias y los artículos de opinión de carácter general están constituyendo su principal ocupación. El profesor Venables tampoco continúa trabajando en temas de la NGE; su actual ubicación académica va unida a un programa sobre temas medioambientales. Quien sí continúa realizando aportaciones a este campo es el profesor M. Fujita, especialmente desde la óptica más teórica y formal. 
a la elaboración de conclusiones sobre las políticas que convendría desarrollar. Ello se justificaba, según Fujita y Krugman (2004), en que el principal objetivo hasta el momento (2003-2004) había sido explicar el porqué se producen las cosas y cómo se producen, pero no si deben ser aceptadas, corregidas o cambiadas.

Lo que acabo de señalar no implica que la aproximación analítica adoptada por la NGE no pueda conducir a extraer conclusiones y a proponer recomendaciones. Lo que señalo es que estas han quedado generalmente excluidas de los análisis de quienes adoptan el enfoque de la NGE, más preocupados siempre por el rigor de la formulación teórica y los modelos que por las cuestiones que deben resolver los responsables de las políticas regionales y urbanas.

A pesar de todo, es cierto que algunos autores adscritos a la NGE reclaman la posibilidad de llevar a cabo intervenciones gubernamentales que corrijan los problemas detectados. Entre otras cosas, porque no se parte -como en el modelo neoclásico más puro- de que el mercado puede resolver las consecuencias que las economías de aglomeración y el esquema centro-periferia prevén para las regiones o aglomeraciones "perdedoras". El propio Krugman (2000) ha sostenido, precisamente, que, puesto que los procesos acumulativos de concentración tienden a producir ganadores y perdedores, quizá a nivel de nación existe un evidente incentivo para que los policy makers procuren asegurar que su país se sitúe como una de las áreas ganadoras. Y otros autores que están en el ámbito de la NGE, como Ottaviano (2011), afirman incluso que si el actual panorama económico no puede entenderse si no se asume algún tipo de imperfecciones del mercado, esto implica necesariamente que no se puede dejar que la responsabilidad de generar una eficiente geografía económica corresponda solo al mercado.

Sin embargo, lo que ha ocurrido con frecuencia es que quienes han trabajado en la elaboración de modelos tipo NGE que se contrastan empíricamente, aunque con restricciones, solo ocasionalmente han entrado en el ámbito de sus implicaciones para las políticas públicas. Ni siquiera en cuanto a si deben o no ser combatidos los efectos no deseables de los procesos de aglomeración (como la congestión y la contaminación que se produce en las grandes concentraciones), o si hay que actuar ante la ausencia de efectos positivos en el caso de las aglomeraciones que son demasiado pequeñas, puesto que la falta de externalidades y de ligazones entre productores dificulta o incluso impide lograr un mayor nivel de actividad.

Desde la óptica de quienes deben diseñar o aconsejar políticas territoriales se ha producido, sin embargo, una situación que es bastante ambigua. Así, aunque se desconfíe de la utilidad de los hallazgos de la NGE para el diseño de políticas, es fácil comprobar que en muchos informes y estudios territoriales publicados en los últimos ańos se incluyen referencias a sus planteamientos, ya sea en apoyo de alguna conclusión o bien para justificar medidas o acciones que se desea emprender. De hecho, varios informes recientes de los grandes organismos internacionales (Banco Mundial, oECD y Comisión Europea, por ejemplo) contienen referencias bastante extensas a los planteamientos de la NGE y a lo que de ellos puede deducirse ${ }^{14}$. Lo

14 Pueden verse, al respecto, tanto el informe How Regions Grow de la oecD (2009) como el del Banco Mundial, Reshaping Economic Geography (The World Bank, 2009) o lo que se recoge en European Commission (2009). 
mismo ocurre con otros informes de consultorías y/o de organismos nacionales o regionales ${ }^{15}$, donde parece que apoyar algunas conclusiones y recomendaciones en lo que han aportado los trabajos de la NGE resulta poco menos que obligado. Hacerlo así supone incorporar argumentos que, por una parte, son más contemporáneos que los que se habían utilizado anteriormente; y por otro, añaden un argumento de autoridad a lo que se expone. Esto último sobre todo en relación con las ventajas y/o desventajas de la aglomeración, el papel de la formación de la fuerza laboral y los costes de transporte, o bien para ilustrar los efectos centrípetos y centrífugos de las relaciones territoriales entre distintas regiones.

Behrens y Robert-Nicoud (2011) han señalado que muchos autores, incluidos los principales promotores de la NGE, se han abstenido y se abstienen de prescribir cualquier recomendación politica. Ello ha dejado la puerta abierta -afirman- para que algunos "pseudocientíficos regionalistas" sean quienes sugieren dichas recomendaciones políticas, con frecuencia sin utilizar ningún criterio explícito de bienestar, ni modelos microeconómicamente bien fundados, ni análisis empíricos. Ambos autores señalan al respecto que los investigadores de la NGE deberían reclamar este terreno como algo que también les es propio. Hace ya varios ańos que Baldwin y otros (2003) denunciaron, precisamente, que la ausencia de implicaciones políticas claras en las aportaciones de quienes adoptan el enfoque de la NGE "exige más trabajo, no menos". Pero esta llamada a la conveniencia de entrar en el ámbito de las políticas apenas ha recibido respuesta: una "anomalía" que clama por un esfuerzo más intenso, y también más comprometido, en el desarrollo de las investigaciones (Behrens \& Robert-Nicoud, 2011).

Aunque lo que acabo de señalar es real, hay que admitir que la NGE ha tenido al menos dos méritos en relación con las políticas públicas. En primer lugar, ha colocado la geografía en el corazón de las relaciones entre el desarrollo y las desigualdades regionales y nos ha recordado que las regiones están interconectadas entre sí y que lo que ocurre en ellas y lo que podría ser conveniente hacer no puede analizarse de forma individual. Y, en segundo lugar, la NGE ha subrayado también la importancia de considerar la estructura espacial de la economía como endógena a la hora de estimar los posibles impactos de las políticas. Ello es muy importante porque invita a tener en cuenta un hecho: los cambios en la estructura espacial de la economía pueden amplificar, embalsar y/o anular incluso los beneficios potenciales que a priori pudieron atribuirse a la realización de unas inversiones y políticas públicas

Algunos trabajos más recientes (Venables, 2007; Behrens, Gaigné \& Thisse, 2009, por ejemplo) han aportado elementos que son muy útiles en relación con las políticas y sus efectos, al señalar, respectivamente, que las economías de aglomeración pueden amplificar los beneficios de las inversiones en transporte urbano, modificando el tamaño de la propia aglomeración, o que los beneficios de la desregulación del sector transporte no pueden considerarse sin tener en cuenta que la

15 Un informe del Tesoro del Reino Unido (2007), otro de la Reserva Federal de Kansas (2006), varios informes de McKinsey, etcétera. 
aplicación de dicha política puede alterar la estructura territorial, provocando resultados distintos de los perseguidos.

Posiblemente donde la NGE está ofreciendo o puede ofrecer más sugerencias en términos de políticas es en el terreno del análisis urbano. En la comprensión, por ejemplo, de los efectos de una política concreta en una determinada aglomeración urbana (ya sea en los transportes, la localización de actividades productivas o en el coste del suelo); así como al obligar a examinar los efectos y relaciones que existen entre los distintos núcleos de una economía y los efectos de las políticas.

R. L. Martin y Sunley (2011) sugieren, precisamente, que los modelos formales y abstractos pueden utilizarse para el análisis de las políticas aplicadas. En primer lugar, porque un modelo permite introducir qué puede ocurrir al ponerlas en práctica y anticipar las "implicaciones políticas" potenciales. Y, en segundo lugar, porque teniendo en cuenta las posibles consecuencias teóricas, los modelos pueden utilizarse para analizar cuestiones políticas "específicas". En este sentido, el modelo centro-periferia de la NGE permite deducir al menos seis implicaciones básicas, que Martin y Sunley (2011) extraen (véase Tabla 1) de las sugerencias realizadas por Baldwin y otros (2003), Ottaviano (2003) y Brackman, Garretsen y Marrewijk (2009). Dichas implicaciones no pueden hacer olvidar, por un lado, los supuestos adoptados como punto de partida y la credibilidad del propio modelo; y por otro, que tales implicaciones tienen que ver con la "posible" solución del modelo desde una posición de equilibrio espacial de la distribución de la actividad económica en relación con otra distinta. En general, lo que se desarrolla son soluciones analíticas en las que lo que se plantean son cuestiones basadas en "qué sucede si" (what if), lo cual puede permitir llevar a cabo análisis bastante sofisticados -aunque no siempre muy realistas ni suficientemente clarospara la toma de decisiones políticas.

\section{CUADRO 1 | Las seis implicaciones políticas básicas del modelo centro-periferia de la NGE}

\begin{tabular}{|l|l|}
\hline \multicolumn{1}{|c|}{$\begin{array}{c}\text { CARACTERÍSTICAS } \\
\text { ANALÍtICAS DEL MODELO }\end{array}$} & \multicolumn{1}{c|}{ IMPLICACIONES PARA LA POLítiCA } \\
\hline Efectos laterales regionales & $\begin{array}{l}\text { Todo tipo de políticas "no regionales" pueden tener consecuencias } \\
\text { para el equilibrio general de la actividad económica. }\end{array}$ \\
\hline Efectos de interacción comercial & $\begin{array}{l}\text { Cambios en el comercio o en la integración económica pueden tener } \\
\text { un alto impacto en el equilibrio espacial de la actividad económica. }\end{array}$ \\
\hline Efectos cerrados $($ lock-in) & $\begin{array}{l}\text { Las políticas temporales pueden tener efectos permanentes sobre el } \\
\text { equilibrio de la distribución espacial de la actividad económica. }\end{array}$ \\
\hline Efectos selectivos & $\begin{array}{l}\text { Las políticas pueden cambiar ("selección" entre) los resultados de } \\
\text { diferentes equilibrios económicos espaciales. }\end{array}$ \\
\hline Efectos coordinación & $\begin{array}{l}\text { Al influir en las expectativas de los agentes, los policy makers pueden } \\
\text { determinar qué estructura de equilibrio espacial se quiere establecer. }\end{array}$ \\
\hline Efectos frontera & $\begin{array}{l}\text { Las políticas necesitan una masa crítica para tener un efecto en el } \\
\text { equilibrio espacial de la distribución de la actividad económica. }\end{array}$ \\
\hline
\end{tabular}

FUENTE SOBRE LA BASE DE BALDWIN Y OTROS (2003) Y BRACKMAN Y OTROS (2009) 


\section{Algunas notas finales}

Como se ha indicado al principio de este trabajo, nuestro objetivo ha sido tratar de ofrecer respuestas a algunas preguntas clave sobre la NGE. Tales respuestas, obviamente, no son "conclusiones", porque dejan abiertas otras posibilidades de interpretación -distintas de las de este autor- sobre lo que significa y lo que ha aportado esta corriente al análisis de las relaciones entre los procesos económicos y el territorio.

A modo de resumen final, parece interesante subrayar algunos puntos e ideas que se han desarrollado a lo largo del texto:

- En el caso de la Economía, la NGe ha servido para poner fin al largo silencio con que la corriente dominante del análisis económico había tratado cuestiones como dónde tiene lugar la actividad económica y cómo evoluciona; por qué tiende a concentrarse; y cuáles son las causas explicativas. Y lo ha hecho sirviéndose de modelos con un soporte analítico-matemático que ha proporcionado mayor solidez y más respetabilidad a algunas tesis e ideas que ya estaban en circulación.

- En el caso de la Geografía Económica tradicional, la irrupción de la NGE ha estimulado el desarrollo de un tipo de aproximaciones mucho más formalizadas y menos descriptivas sobre algunos temas que ya se venían estudiando, como la concentración de la población y de la actividad económica, la formación de cinturones industriales o agrarios, o la localización de los servicios, entre otros.

- Como se ha expuesto, cabe cuestionarse sin embargo la "novedad" de la NGE. No, quizá, desde la perspectiva metodológica, donde sí existe un claro componente novedoso; pero sí al tener en cuenta numerosas aportaciones que los líderes de la NGE ignoraron desde el principio, para calificar sus conceptos y algunos desarrollos como "nuevos". Piénsese en algunas aportaciones a la teoría de la localización o sobre las áreas de mercado, así como las aproximaciones micro- y macro- al análisis regional. Nombres como Christaller, Lösch, Hoover, Alonso o Isard, por citar solo un reducido número de ellos, prácticamente no aparecen nunca citados en los trabajos de la primera etapa de la NGE. Algo parecido sucede con los geógrafos económicos más renombrados de la línea convencional.

- Por otra parte, bastantes de los conceptos que la NGE colocó en una posición muy destacada eran ya bien conocidos con anterioridad. Hay que releer a Marshall, a Myrdal, a Hirschman o a Perroux, entre otros, para constatar la certeza de esta afirmación y los desarrollos que ellos y otros aportaron a los conceptos de aglomeración, economías externas, ligazones hacia adelante y hacia atrás de las industrias, etcétera. En los modelos centro-periferia de la NGE, la "novedad" está más en el grado de abstracción y en el tratamiento conjunto de las distintas fuerzas que operan (centrífugas y centrípetas), que en el "descubrimiento" de auténticas nuevas ideas.

- El desarrollo de la NGE se ha caracterizado por la construcción de modelos, lo que exige -como es sabido- aceptar determinados supuestos y simplificaciones 
como punto de partida. El propósito de "liberar" posteriormente dichas restricciones introduciendo un mayor grado de realismo no se ha cumplido con excesiva amplitud. Como se subrayó en la cuarta sección, dentro de la propia corriente de la NGE han surgido en los últimos años numerosas críticas sobre el carácter limitado que muestran muchos de los modelos producidos. En bastantes casos solo consideran cómo se comporta un factor, o los movimientos entre dos posibles localizaciones alternativas, o qué sucede cuando se produce un determinado cambio, "permaneciendo todo lo demás igual". El valor de estos esfuerzos no es despreciable, por supuesto, pero con frecuencia se detienen en la frontera del análisis teórico o estrictamente académico.

- Si bien hay excepciones, un rasgo muy claro de la NGE es su escaso interés por entrar en el terreno de las políticas que sería deseable llevar a cabo para frenar, incentivar, compensar, etcétera, los procesos que estudian sus modelos. Hacer propuestas operativas de política económica general o de política regional y urbana es algo que está muy ausente en los trabajos de quienes lideran y participan en el desarrollo de la NGE. Ello, sin embargo, no es óbice para que algunos organismos internacionales, consultores y entidades responsables del desarrollo territorial incluyan en sus informes citas y referencias a la NGE. Con frecuencia lo hacen buscando el "apoyo" de teorías que se considera que están "à la page”, aunque sin una clara relación con los problemas reales examinados o con sus posteriores recomendaciones.

- La NGE se encuentra en una fase de clara "madurez", lo que no siempre suele ir acompañado de innovaciones importantes. Krugman ya señaló hace años que la euforia con que en un principio se acogieron las ideas de la NGE ha ido dejando paso a algunas críticas, permitiendo considerar incluso que las "nuevas" o las más recientes aportaciones no son particularmente innovadoras. Esto respondería al esquema que con frecuencia caracteriza a la investigación económica: el ciclo expansión-caída, más o menos dilatado en el tiempo.

- Uno de los problemas que también hay que abordar es que los modelos de la NGE son, en bastantes casos, demasiado simples, demasiado estilizados para representar o reproducir bien la geografía económica real. De hecho, como se ha subrayado en el texto, son varios los autores -incluido quien suscribe este trabajo- que han coincidido en reclamar un mayor esfuerzo para que los modelos sean cada vez más realistas y para que la teoría vaya siempre acompańada de análisis empíricos y de casos, incluyendo no solo sugerencias sobre los resultados, sino indicaciones y alternativas para la solución de los problemas detectados. 


\section{Referencias bibliográficas}

Alonso, W. (1964). Location and land use: Toward a general theory of land rent. Cambridge, MA: Harvard University Press.

Baldwin, R. E. (1998). Agglomeration and endogenous capital. Discussion Paper, 1847. London: Centre for Economic Policy Research (CEPR).

Baldwin, R. E. (1999). Agglomeration and endogenous capital. European Economic Review, 43, 253-280. Recuperado de http://bit.ly/JKvkiz

Baldwin, R. E., Forslid, R., Martin, P. H, Ottaviano, G. I. P. \& Robert-Nicoud, F. L. (2003). Economic Geography and Public Policy. Princeton, NJ: Princeton University Press.

Behrens, K. \& Robert-Nicoud, F. (2011). Tempora mutantur: in search of a new testament for NEG. Journal of Economic Geography, 11(2), 215-230. doi: 10.1093/jeg/lbq039

Behrens, K \& Thisse, J-F. (2007). Regional economics: A new economic geography perspective. Regional Science \& Urban Economics, 37(4), 457-465.

Behrens, K., Gaigné, C. \& Thisse, J-F. (2009). Industry location and welfare when transport costs are endogenous. Journal of Urban Economics, 65(2), 195-208.

Bhattacharjea, A. (2010). Did Kaldor anticipate New Economic Geography? Yes, but... Cambridge Journal of Economics, 34(6), 1057-1074.

Blaug, M. (1986). Great Economists before Keynes. Cambridge and New York: Cambridge University Press.

Brakman, S., Garretsen, H. \& Marrewijk, C. (2009). The New Introduction to Geographical Economics. Cambridge, MA: Cambridge University Press.

Bunge, M. (1997). La Ciencia, su método y su filosofía. Buenos Aires: Sudamericana.

Bunge, W. (1966). Theoretical Geography. Lund: The Royal University of Lund.

Christaller, W. (1933). Die zentrale Orte in Süddeutschland. Jena: G.Fischer.

Cuadrado-Roura, J. R. (Dir.) (2010). Politica Económica. Elaboración, objetivos e instrumentos (4a ed.). Madrid: McGraw-Hill.

David, W. L. (1988) Political Economy and Economic Policy. N York and London: Praeger.

Duverger, M. (1964). Introduction à la politique. Paris: Gallimard.

Englmann, F. C. \& Walz, U. (1995). Industrial centers and regional growth in the presence of local inputs. Journal of Regional Science, 35(1), 3-27.

European Commission (2009). Paul Krugman's New Economic Geography: Past, present and future. Bureau of Economic Policy Advisors Seminar, en: http://ec.europa.eu/bepa/ expertise/seminars/index_en.htm

Fingleton, B. (2011). The empirical performance of the NEG with reference to small areas. Journal of Economic Geography, 11(2). 267-279. doi: 10.1093/jeg/lbq044

Fujita, M. \& Krugman, P. (1995). When is the economy monocentric? Von Thünen and Chamberlain Unified. Regional Science and Urban Economics, 25, 505-528. Recuperado de http://bit.ly/1iWH0NL

Fujita, M. \& Krugman, P. (2004). La nueva geografía económica: pasado, presente y futuro. Investigaciones Regionales, 4. Recuperado de http://www.redalyc.org/ pdf/289/28900409.pdf [Original: The new economic geography: Past, present and the future. Papers in Regional Science, 83(1), 139-164, 2004. doi: 10.1007/s10110003-0180-0]. 
Fujita, M. \& Thisse, J-F. (2002). Economics of agglomeration. Cities, Industrial Location, and Regional Growth. Cambridge, MA: Cambridge University Press. Recuperado de http:// bit.ly/K8FoRP

Fujita, M. \& Thisse, J-F. (2009). New Economic Geography: an appraisal on the occasion of Paul Krugman 2008 Nobel Prize in Economic Sciences. Regional Science and Urban Economics, 39(2), 109-119.

Fujita, M., Krugman, P. \& Venables, A. (1999). The spatial economy. Cities, regions and international trade. Cambridge, MA: The MIт Press. Versión en espańol: Economía espacial. Las ciudades, las regiones y el comercio internacional. Barcelona: Labor, 2000.

Hagget, P. (1965). Locational analysis in Human Geography. London: E. Arnold.

Hirschman, A. O. (1958). The strategy of economic development. New Haven, ст: Yale University Press.

Hoover, E. (1959). Anatomy of a metropolis. Harvard University Press.

Isard, W. (1956). Location and Space-Economy. Cambridge, MA: The міт Press.

Krugman, P. R. (1991). Increasing returns and economic geography. Journal of Political Economy, 99(3), 483-499. Recuperado de http://bit.ly/1hzMtd5

Krugman, P. R. (1993a). On the relationship between trade theory and location theory. Review of International Economics, 1(2), 110-122. doi: 10.1111/j.1467-9396.1993.tb00009.x

Krugman, P. R. (1993b). First nature, second nature and metropolitan location. Journal of Regional Science, 33(2), 129-144. doi: 10.1111/j.1467-9787.1993.tb00217.x

Krugman, P. R. (2000). Where in the world in the "New Economic Geography". En G. L. Clark, M. P. Feldman \& M. S. Gerler (Coords.), The Oxford Handbook of Economic Geography (Cap. 3). Oxford: Oxford University Press.

Kuhn, T. S. (1962). The structure of scientific revolutions. Chicago, Il: University of Chicago Press.

Lloyd, P. \& Dicken, P. (1972). Location in space: A theoretical approach to Economic Geography. New York: Harper \& Row.

Lösch, A. (1954). The Economics of Location. New Haven, CN: Yale University Press. Versión inglesa de la primera edición en alemán: Die räumliche Ordnung der Wirtschaft, de 1940.

Marshall, A. (1890). Principles of Economics. London: Macmillan.

Martin, Ph. \& Ottaviano, G. I. P. (1999). Growing locations: industry location in a model of endogenous growth. European Economic Review, 43, 281-302. Recuperado de http:// web.cenet.org.cn/upfile/7531.pdf

Martin, Ph. \& Ottaviano, G. I. P. (2001). Growth and agglomeration. International Economic Review, 42(4), 947-968. doi: 10.1111/1468-2354.00141

Martin, R. L. (2010). The New Economic Geography: credible models of the economic landscape? En R. Lee, A. Leyshon, L. McDowell \& P. Sunley(Eds.), A Compendium of Economic Geography (pp. 53-73). London: Sage. doi: http://dx.doi. org/10.4135/9781446201114.n4

Martin, R. L. \& Sunley, P. (1996). Paul Krugman's geographical economics and its implications for regional development theory. A critical assessment. Journal of Economic Geography, 72(3), 259-292.

Martin, R. L. \& Sunley, P. (2011). The new economic geography and policy relevance. Journal of Economic Geography, 11(2), 357-369. doi: 10.1093/jeg/lbq042

Morril, R. (1970). The spatial organization of society. Belmont, CA: Wadsworth. 
Myrdal, G. (1953). The political element in the development of economic theory. London: Routledge \& Kegan Paul. Versión en español: El elemento politico en el desarrollo de la teoría económica. Madrid: Gredos, 1967.

Myrdal, G. (1957). Economic theory and underdeveloped regions. London: Duckworth.

Organisation for Economic Co-operation and Development (oECD). (2009). How Regions Grow: Trends and analysis. París: Autor.

Ottaviano, G. I. P. (2003). Regional policy in the global economy: insights from the new economic geography. Regional Studies, 37, 665-673.

Ottaviano, G. I. P. (2011). 'New' new economic geography: firm heterogeneity and agglomerations economies. Journal of Economic Geography, 11(2), 231-240. doi: $10.1093 / \mathrm{jeg} / \mathrm{lbq} 041$

Pareto, W. (1920). Fatti e teorie. Florencia: Vallechi.

Perroux, F. (1955). Note sur la notion de pôle de croissance. Économie Appliquée, 8, 307-320.

Puga, D. (1999). The rise and fall of regional inequalities. European Economic Review, 43(2), 303-334.

Puga, D. \& Venables, A. J. (1996a). The spread of industry: Spatial agglomeration in economic development. Journal of Japanese and International Economies, 10(4), 440-464. Recuperado de http://bit.ly/1hzRItk, como Discussion Paper n. ${ }^{\circ} 279$.

Puga, D. \& Venables, A. J. (1996b). Preferential trading arrangements and industrial location. Journal of International Economics, 43, 347-368. Recuperado de http://bit. ly/1 euLQfB, como Discussion Paper n. ${ }^{\circ} 267$.

Puga, D. \& Venables, A. J. (1999). Agglomeration and economic development: import substitution vs. trade liberalization. Economic Journal, 109(455) (Apr.), 292-311.

Richardson, H. W. (1975). Elementos de economía regional. Madrid: Alianza.

Rojas López, J. J. (2009). Las dimensiones espaciales del Informe 2009 del Banco Mundial: otra "nueva geografía" sin territorio. Revista Geográfica Venezolana, 50(2), 331-342. Recuperado de http://bit.ly/1ajoavU

Schlefer, J. (2012). The assumptions economists make. Cambridge, MA and London: The Belknap Press of Harvard University Press.

Sheppard, E. (2011). Geographical political economy. Journal of Economic Geography, 11(2), 319-331. doi: 10.1093/jeg/lbq049

Storper, M. (2011). Why do regions develop and change? The challenge for geography and economics. Journal of Economic Geography, 11(2), 333-346. doi: 10.1093/jeg/lbq033

Venables, A. J. (1996). Equilibrium locations of vertically linked industries. International Economic Review, 37(2), 341-359.

Venables, A. J. (2007). Equilibrium locations of vertically linked industries. Journal of Transport Economics and Policy, 41, 173-188.

World Bank. (2009). Reshaping economic geography. World development report 2009. Washington, D.C.: The International Bank for Reconstruction and Development (IBRD) / The World Bank. Recuperado de http://bit.ly/1lYbAE7 